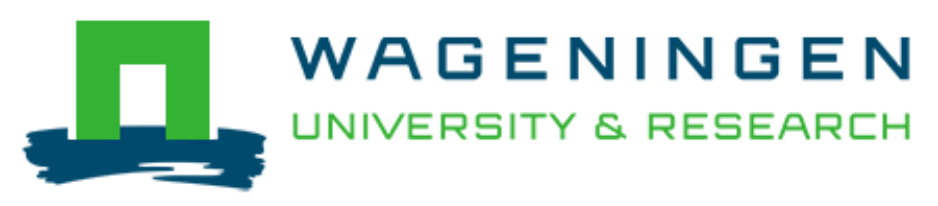

Heart rate and skin conductance responses to taste, taste novelty, and the (dis)confirmation of expectations Verastegui-Tena, L., van Trijp, H., \& Piqueras-Fiszman, B.

This is a "Post-Print" accepted manuscript, which has been published in "Food Quality and Preference"

This version is distributed under a non-commercial no derivatives Creative Commons

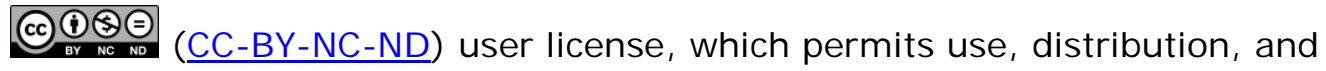
reproduction in any medium, provided the original work is properly cited and not used for commercial purposes. Further, the restriction applies that if you remix, transform, or build upon the material, you may not distribute the modified material.

Please cite this publication as follows:

Verastegui-Tena, L., van Trijp, H., \& Piqueras-Fiszman, B. (2018). Heart rate and skin conductance responses to taste, taste novelty, and the (dis)confirmation of expectations. Food Quality and Preference, 65, 1-9. DOI:

10.1016/j.foodqual.2017.12.012

You can download the published version at:

https://doi.org/10.1016/j.foodqual.2017.12.012 


\title{
Heart rate and skin conductance responses to taste, taste novelty, and the (dis)confirmation of expectations
}

Luz Verastegui-Tena, Hans van Trijp, Betina Piqueras-Fiszman*

betina.piquerasfiszman@wur.nl

Marketing and Consumer Behaviour Group, Wageningen University and Research Centre, Hollandesweg 1, 6706 KN

Wageningen, The Netherlands

Marketing and Consumer Behaviour Group, Wageningen University and Research Centre, Hollandesweg 1, 6706 KN

Wageningen, The Netherlands

*Corresponding author.

\begin{abstract}
It is unclear whether the responses of the autonomic nervous system (ANS) can measure how people respond to food. Results focused on emotional responses are contradictory; therefore, the focus has shifted to other components of emotion, such as appraisals. The aim of this study was, therefore, to evaluate the differences in ANS responses related to appraisals; particularly taste novelty, valence, and the disconfirmation of expectations. A hundred and fifty-five participants joined this study. They tasted samples of different valence (sweet and bitter) twice: the first time without knowing the taste and the second while being informed of the taste. After this first block, participants tasted two additional samples: one that confirmed expectations and one that disconfirmed them. Heart rate and skin conductance were measured. Results show that the second experience with a taste led to cardiac deceleration. Heart rate changes were only related to valence when participants' expectations were (dis)confirmed. Heart rate decreased for those tastes that disconfirmed expectations and increased for those that confirmed them and the sweet sample had larger increases in heart rate than the bitter. Skin conductance changed in regards to novelty and valence but not to the disconfirmation of expectations. It increased for the bitter sample, decreased for the sweet, and was always higher during the first experience than during the second. In conclusion, the results suggest that cardiac responses are more sensitive to novelty and the disconfirmation of expectations while skin conductance responses capture novelty and valence.
\end{abstract}

Keywords: Autonomic nervous system; Heart rate; Skin conductance; Taste; Novelty; Expectations 


\section{Introduction}

The acceptance and rejection of food are influenced by a series of variables. While sensory liking plays a role, other variables such as environmental factors (ambiance and food varieties), the presence of others, or even the emotions felt are of importance. Emotions have been found to have a bidirectional effect with food: they influence food choice and the foods consumed can influence them (He, Boesveldt, de Graaf, \& de Wijk, 2016). Food researchers have therefore developed an interest in the subject of emotional responses to food.

Researchers have been looking for a reliable way to measure how people instinctively respond to food products (Mojet et al., 2015). Explicit measurements such as self-reports are commonly used but have significant disadvantages. Variables such as social desirability may bias participants and make them enhance positive aspects or deny negative ones when reporting their reactions. Moreover, reporting one's emotional state may lead to reflective processes that can change the emotional experience (Bartoszek \& Cervone, 2016). For these reasons, along with the fact that emotions are said to have a conscious and an unconscious component, the additional use of implicit measurements (non-verbal) is increasing. Implicit measurements reflect uncontrollable fast mechanisms that the person is unaware of (Lebens et al., 2011). They may provide a more holistic view regarding the emotional and motivational responses to food (Walsh, Duncan, Bell, O'Keefe, \& Gallagher, 2017). Commonly used implicit measurements in food research include facial expressions, neuroimaging or physiological measures, particularly those of the autonomic nervous system (ANS) (Spinelli \& Niedziela, 2016).

The ANS is a component of the peripheral nervous system which is in charge of both the activation (sympathetic branch) and relaxation (parasympathetic branch) of the body (Mauss \& Robinson, 2009). It functions without voluntary control or awareness of the person (McCorry, 2007). While the ANS is considered a major component of the emotional response, there are still more controversies than consensual views on the topic of ANS and emotion (Levenson, 2014). The ANS activity is not exclusively a function of emotional responding but encompasses a variety of other functions such as homeostasis, effort, and attention. This makes it difficult for researchers to determine whether the ANS is a good measure of emotional responses to food. Results regarding the link of ANS and emotion are inconsistent among studies. The focus has, therefore, shifted to the study of ANS and other dimensions or components of emotion that might lead to clearer results than emotions alone (Mauss \& Robinson, 2009). One such component could be appraisals.

Appraisals, same as the ANS, are automatic and unconscious (Ellsworth \& Scherer, 2002). They could be seen as applying a series of "checks" and "sub-checks" when perceiving a stimulus. The stimulus is defined as relevant by a series of dimensions which are part of the main structure in which the evaluation takes place. Once the stimulus is seen as relevant, further appraisals occur. These appraisals eventually lead to an emotion and to ANS responses. Hence, the ANS responses created by emotions are intimately related to the appraisals that produced them (Derryberry \& Reed, 2002; Frijda \& Mesquita, 1998).

The most basic appraisals at the level of perception and of importance for food research are those of novelty and valence or intrinsic pleasantness. Novelty detection draws attention and leads to further processing to determine whether any action is required (Ellsworth \& Scherer, 2002). Novelty can be extracted through perceptual processing. In general, situations that are novel are situations in which the person has not had any previous experience. Most situations, however, are not completely novel. Stimuli are compared to pre/existing ideas or knowledge (Lazarus \& Folkman, 1984). In the case of food, for example, people already have preconceived ideas about the sensory characteristics of the product (taste, texture, among others) and how much they are going to like it (Tarancón, Sanz, Fiszman, \& Tárrega, 2014). Hence, some appraisal theories do not consider novelty but instead include related concepts such as disconfirmation of expectations or unexpectedness to capture this incongruency between existing stimuli and the expectations of the person (Derryberry \& Reed, 2002; Moors, Ellsworth, Scherer, \& Frijda, 2013). The appraisal of valence determines which will be the reaction or response of the organism. These reactions could be approach (led by attraction and liking) or avoidance (led by aversion or disgust). The appraisal of valence usually happens quickly and, as a result, is difficult to separate from that of the experience of attention. Particularly in those cases in which the stimulus is seen as negative, other appraisals follow such as certainty, agency, and power (Ellsworth \& Scherer, 2002).

Literature regarding ANS, novelty, and valence in food research have used different types of stimuli and shown different results. Rousmans, Robin, Dittmar, and Vernet-Maury (2000) found that compared to baseline, a sucrose solution led to the smallest changes in ANS responses, including heart rate (instantaneous heart rate) and electrodermal activity (skin resistance amplitude index and ohmic perturbation duration index), while a bitter solution led to the largest changes. Danner, Haindl, Joechl, and Duerrschmid (2014) found that, compared to baseline, juices that were disliked (and therefore had a negative valence) were associated with higher skin conductance responses (SCR) than those that were liked (and 
therefore had a positive valence) but no differences were seen in HR. Likewise, de Wijk, Kooijman, Verhoeven, Holthuysen, and de Graaf (2012) found that disliked foods led to a decrease in SCR in adults compared to liked foods but to similar heart rate responses between liked and disliked foods. These studies, however, do not consider the differences in ANS when a stimulus is encountered for the first time, and consequently seen as novel. Worth mentioning is the study by Delplanque et al. (2009) which looks at the sequential unfolding of novelty and pleasantness in odors. The results of this study show that novel odors elicited greater SCR amplitudes than odors that were repeated and that negative (unpleasant) odors led to greater SCR amplitudes than positive (pleasant) odors. In contrast, novel odors led to a decrease in heart rate compared to repeated odors and positive odors led to a stronger cardiac deceleration than negative odors. Studies regarding ANS and the disconfirmation of expectations in food research are scarce. Data by de Wijk and Boesveldt (2016) show that when participants tasted a breakfast drink that was different from the one that was visually inspected, the ANS responses, particularly that of skin conductance, were increased. Another study (Verastegui-Tena, SchulteHolierhoek, van Trijp, \& Piqueras-Fiszman, 2017) showed that ingredient images that disconfirmed expectations from the tasted experience led to cardiac deceleration regardless of the valence of the image while in the case of skin conductance only the negative image led to an increase that differed from that of a neutral congruent image.

It seems that the use of ANS responses in food research, though a novel approach in the area, needs a deeper understanding in order to be used adequately. Disentangling the responses to basic appraisals can be the tool to a better understanding and interpretation. To the best of our knowledge, no study has looked at the ANS responses to novelty, valence, and the disconfirmation of expectations together in a systematic way. The aim of this study is, therefore, to discern the differences in ANS responses related to novelty (first encounter with a taste with no previous information), to the disconfirmation of expectations, and to valence. For this purpose, the experiment was divided in two blocks. In the first block, participants drank two times a sample with a positive taste and a sample with a negative taste (these tastes being pretested for valence). The first time without being told beforehand the taste of the sample and the second time with previous knowledge of the taste of the sample. The effect of novelty related to the first experience with a taste (henceforth referred to as "taste novelty") was measured by comparing the changes from baseline of the ANS responses of the first time participants tasted the sample to those of the second time. Valence (henceforth referred to as "taste" or "taste valence") was assessed by comparing the changes from baseline of the ANS responses associated to the positive taste to those of the negative one. For the second block of the study, participants tasted samples with a taste that confirmed their expectations (the taste matched the one they had been told beforehand and was, therefore, expected) and a taste that disconfirmed them (the taste was different to that they had been told beforehand and was, therefore, unexpected). The effect of the disconfirmation of expectations was assessed through the comparison of the changes from baseline of the ANS responses between the expected sample and the unexpected one.

Our hypotheses were that (1) the novelty of the taste would engage the attention of the participant and, as a consequence, the first tasting would lead to larger increases in skin conductance and smaller decreases in heart rate than the second tasting; (2) following the pattern of previous studies, the positive taste will lead to lower ANS responses than the negative taste; and (3) samples that disconfirm expectations will activate a defensive response and, therefore, lead to higher increases in skin conductance and heart rate.

\section{Materials and methods}

\subsection{Screening and selection of participants}

A hundred and fifty-five participants ranging from 18 to 45 years of age were recruited from the Wageningen area and surroundings. Participants were recruited via flyers and advertisements in buildings and supermarkets close to Wageningen University. They were excluded if they had a BMI higher than $27.5 \mathrm{~kg} / \mathrm{m} 2$, had any known cardiac problems, were non-tasters for bitter or were allergic to the ingredients of the stimuli used. In order to test if they were non-tasters for bitter, all potential participants were given a phenylthiocarbamide (P.T.C) test paper (Selfcontrol CD 265 D 01, Bern, CH) and asked to chew it. Those who could not sense the bitterness of the P.T.C test paper were given a sample with quinine to confirm their taster status. Participants who could sense the bitterness were given a summary of the procedure that would be followed during the study and were asked to schedule an appointment if they fulfilled all requirements and agreed to participate. The Social Sciences Ethics Committee of Wageningen University approved the study.

\subsection{Experiment session}


The study took place in the Marketing and Consumer Research group premises from Wageningen University, the Netherlands. Participants were instructed to wear comfortable clothes for the study (sport clothes were recommended). The study took place in a well-lit white room where participants were seated in front of a computer with OpenSesame version 3.1.2 (Mathôt, Schreij, \& Theeuwes, 2012). Before starting the test, they were given an informed consent. Once the participant had signed the consent, the researcher started placing the sensor pads.

Seven sensor pads (Kendall ${ }^{\mathrm{TM}}$ H98S8 $60 \mathrm{~mm}$ micropore ECG electrodes) were placed on each participant. Five were on the chest and two on the back. For the measurement of skin conductance, two sensors were placed (Biopac $®$ TSD 203 Electrodermal Response Transducer), one on the index finger and the other on the middle finger of the non-dominant hand. The researcher checked all signals to avoid any problems due to electrode misplacements. After all signals were checked, participants were asked to remain still, to close their eyes, and to breathe normally for one minute.

The study consisted of two blocks. The first block involved two tasting rounds of three samples and was used to test the effect of taste novelty and taste (Fig. 1). For this part, participants were separated randomly in three groups. For all groups, the first sample of each round was water and was used as a baseline. The second sample was given without giving the participants any previous information of the taste. The third sample was identical to the previous one and was given after participants were told the taste. The second and third samples could be either sweet (positive valence) or bitter (negative valence). Participants in Group 1 were given bitter samples in the first round and sweet samples in the second round. For the participants in Group 2 the order was altered, they received the sweet samples in the first round and the bitter ones in the second. At the end of each tasting, participants were directed to a questionnaire in which they rated, by means of a $100 \mathrm{~mm}$ visual analogue scale (VAS), the pleasantness and taste intensity of the bitter and sweet samples. Group 3 was given water samples using a similar procedure but without asking participants to rate the pleasantness and taste intensity of the water. This was done to avoid any confusion or suspicion of the participants, as water is considered to be neutral. Group 1 and 2 were used for the main analysis of taste novelty and pleasantness. Due to the lack of pleasantness and taste intensity ratings, Group 3 could only be used for a separate analysis to have a general overview of how neutral samples compare to those with taste.

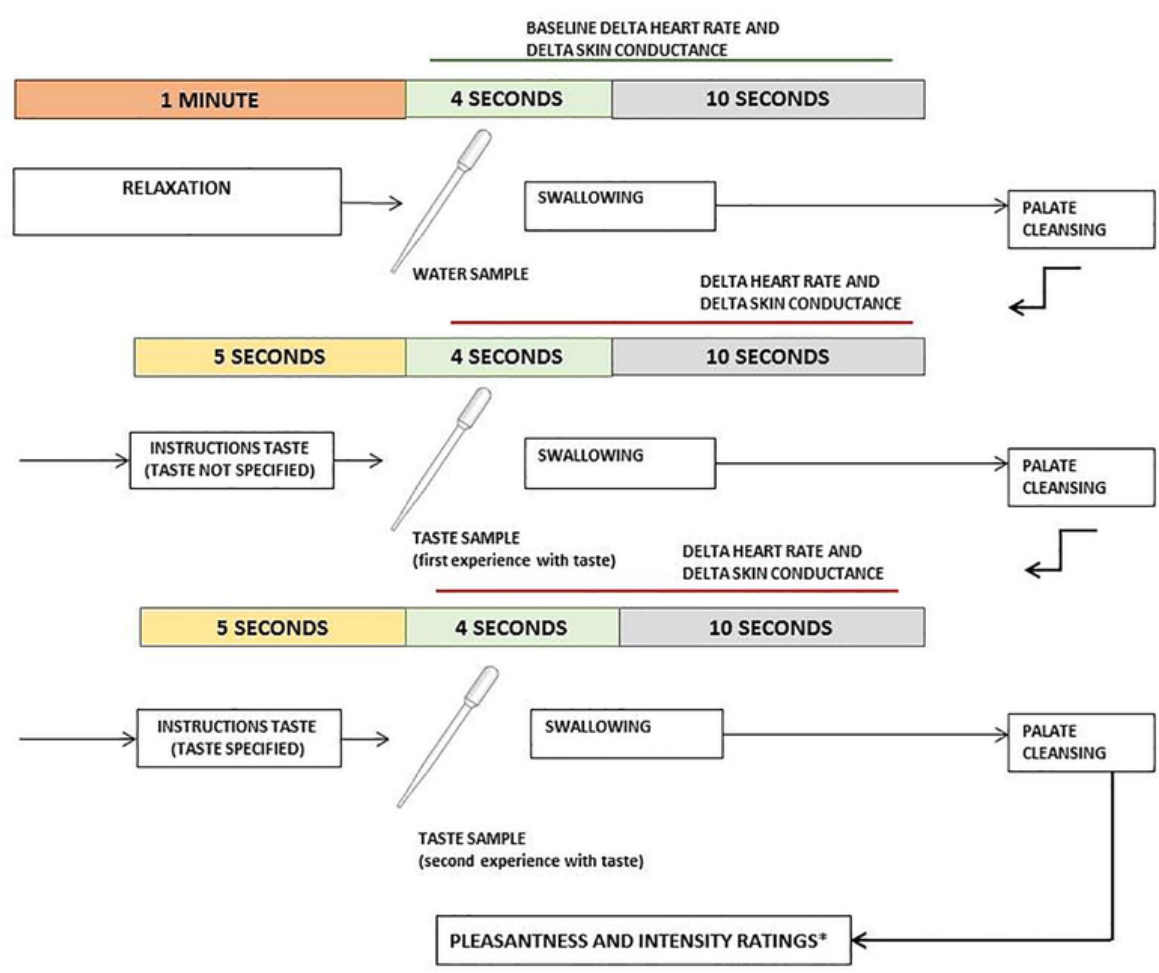

Fig. 1 Procedure of the first block of the study (one round of three tastings). Heart rate and skin conductance were measured while participants tasted twice sweet and bitter samples. The first sample consisted of water and served as a baseline. The second and third samples contained the same taste. Participants tried the second sample without previous knowledge of the taste (taste not specified). For the third sample participants were told beforehand the taste (taste specified).

The second block of the study involved a tasting of four samples and was used to test the effect of the disconfirmation of expectations (Fig. 2). For this part, participants were divided in five groups. For all groups, the first and third samples were water. The second and fourth samples varied according to the group. For Group 1, participants were told that the second 
and fourth samples were bitter, but in reality the fourth sample was sweet, an unexpected taste that disconfirmed expectations. For Group 2 the order was reversed, the unexpected sweet sample was given in the second position. Groups 3 and 4 were told that both the second and fourth samples were sweet. However, for Group 3 the fourth sample was in reality bitter, while for Group 4 the order was reversed and the unexpected bitter sample was given in the second position. All tastings where the opposite taste was given were covered as a mistake from the researcher giving the wrong sample. Finally, to be able to compare the taste samples with a neutral sample, Group 5 received water four times with no manipulation of the taste expectations of the samples.

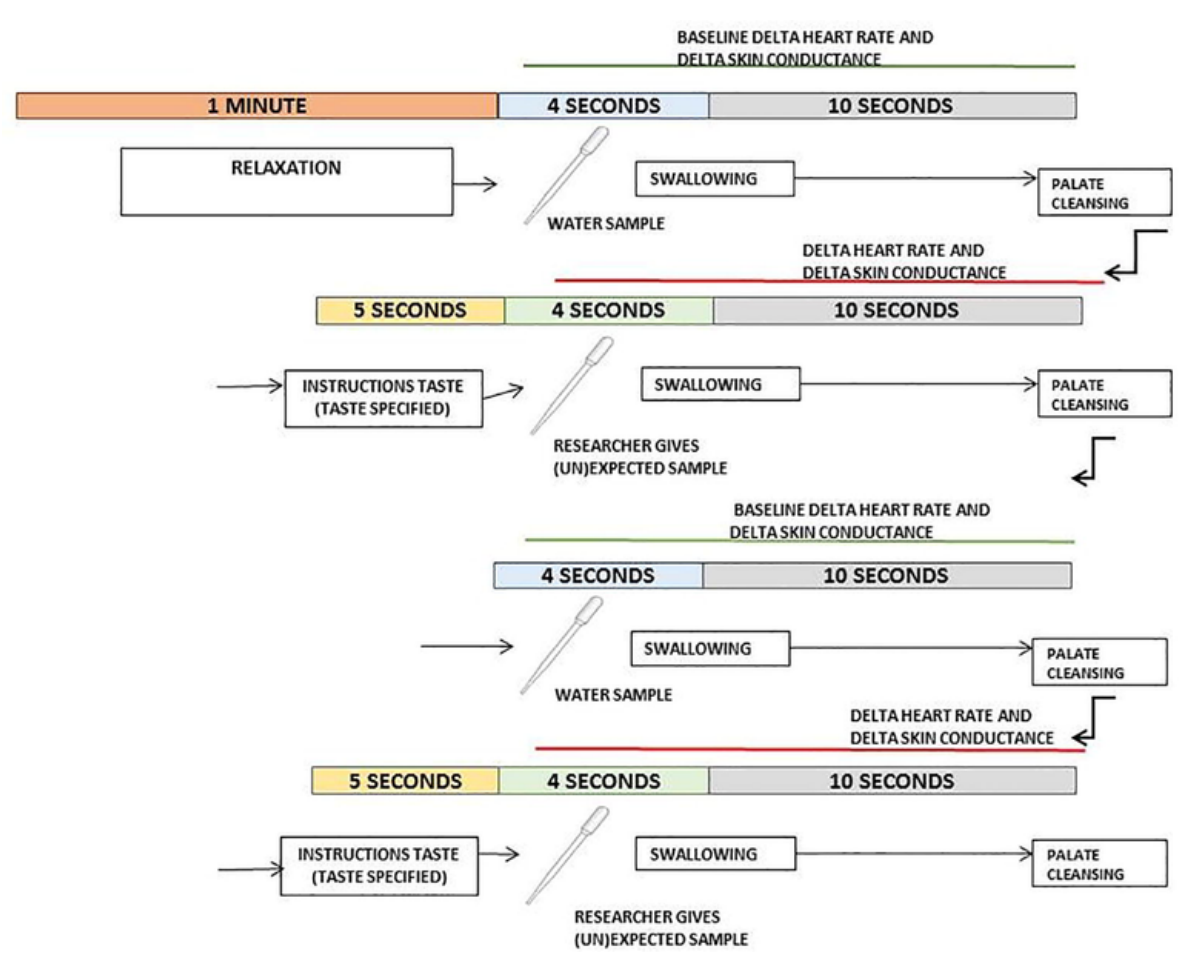

Fig. 2 Procedure of the second block of the study. Heart rate and skin conductance were measured while participants tasted samples that confirmed (expected taste) and disconfirmed (unexpected taste) expectations. The first and third samples consisted of water. The second and fourth samples could have either an expected or unexpected taste, depending on the group the participant was assigned to.

Participants were asked to cleanse their palate with water after each sample and to make sure the taste was gone from their mouth before continuing with the next part of the test. After finishing the test, participants were debriefed. They were told the reason why they were given a sample different from the one they were told and the main objectives of the study.

\subsection{Tasting samples}

The samples used in this study were a sample with a positive valence consisting of sucrose (sweet) and a sample with a negative valence consisting of quinine (bitter). The sucrose sample had a molarity of $4.5 \times 10-1 \mathrm{M}$. For its preparation, $154.033 \mathrm{~g}$ of table sugar (Van Gilse Krystal Suiker, Suiker Unie, Oud Gastel NL) were added in $1 \mathrm{~L}$ of water. The quinine sample had a molarity of $7.5 \times 10-5 \mathrm{M}$. For its preparation $0.0297 \mathrm{~g}$ of quinine monohydrochloride dihydrate $>95 \% \mathrm{FG}$ (Sigma Aldrich Flavours \& Fragrances, Milwaukee USA) were added in $1 \mathrm{~L}$ of water. The concentration of the samples was determined in preliminary tests done to ensure that the taste was detectable by participants and that the bitter sample was considered unpleasant (and hence, had a negative valence) in comparison to the sweet one (pleasantness ratings in preliminary tests of $58.4 \pm 18.0$ in a $100 \mathrm{~mm}$ VAS scale for the sucrose solution and $23.4 \pm 15.1$ for the bitter solution). All samples were presented at room temperature and given with a $3 \mathrm{ml}$ plastic pipette to avoid any movement from the participant. The volume given to each participant was $2 \mathrm{ml}$. This was chosen to imitate the normal swallow of saliva, which ranges from 1 to $2 \mathrm{ml}$, and to avoid big swallowing motions due to the size of the bolus (Levine, Ramchandani, \& Rubesin, 2012).

\subsection{Physiological measurements}


Heart rate and skin conductance were measured throughout the study. For these measurements the VU-AMS version 3.9 (de Geus, Willemsen, Klaver, \& van Doornen, 1995) was used. The ECG had a sampling rate of $1000 \mathrm{~Hz}$ and heart rate was obtained from the time between two adjacent $\mathrm{R}$ waves. Skin conductance was sampled at a rate of $10 \mathrm{~Hz}$ with a signal range between 0 and $95 \mu \mathrm{s}$. The signal was filtered both in forward and reverse direction with a low pass filter with a cut off frequency of $2 \mathrm{~Hz}$.

\subsection{Data treatment and analysis}

The VU DAMS program (version 3.9) was used for the extraction of the heart rate and skin conductance responses. All data was inspected for artifacts and labelled. The deviation from the baseline for heart rate (delta heart rate) and skin conductance (delta skin conductance) of each tasting was calculated by subtracting the average heart rate and skin conductance of the tasting of the water samples to the average heart rate and skin conductance of the samples with taste. For the statistical analysis the R software version 3.4.0 was used.

To test if taste and taste novelty had an effect on ANS responses, heart rate and skin conductance from the first block of the study were analyzed by means of a mixed model anova stating subject as random factor, the variables taste (sweet or bitter), taste novelty (first or second experience with the taste), and as well as the interactions between the taste and taste novelty as fixed factors. Taste intensity ratings were also included as a covariate. To confirm that the sweet and bitter samples differed in pleasantness, the pleasantness ratings of the sweet and bitter samples were compared with a pairedsample t-test.

To test if the disconfirmation of expectations had an effect on ANS responses, heart rate and skin conductance from the second part of the study were analyzed by means of a mixed model anova considering subject as random factor and the variables real taste (the actual taste of the sample), disconfirmation (confirmation or disconfirmation of the taste told to participants) as well as the interactions between the real taste and the disconfirmation as fixed factors.

Two supplementary analyses were done to corroborate the results found. The first analysis was done with the responses of the first block of the study. The ANS responses of the two tastings of the bitter, sweet, and water groups were assessed. A mixed model with subject as random factor and the variables taste (sweet, bitter or water), taste novelty (first or second experience with the taste) and the interaction between taste and taste novelty was done for each taste. As participants in the water group did not rate the pleasantness and taste intensity of the sample, the covariate taste intensity was not included in the model. The assumption of this analysis was that the effect of taste novelty will be present in all samples and that the effects of taste will make the responses stronger for the sweet and bitter samples than for the water samples. The second analysis was done with the responses of the samples of the second block of the study. The ANS responses for the samples given in the second position were used. Only the bitter and sweet samples that confirmed expectations and the water samples were analyzed (note that the group that received water were not deceived at any moment, hence the use of the confirmation trials for the taste samples). An anova with the variable real taste (actual taste of the sample) was used. The assumption was that the responses for the water samples should be of a smaller magnitude than the other samples with taste and that the responses between the samples with taste will be similar to those of the main analysis.

For all the models used in the analysis, the variables gender, age, and BMI were assessed in separate models and only added to the main one if their inclusion affected the general outcome of the model. Post hoc analyses were performed using Tukey's honest significance test (Tukey's HSD) for multiple comparisons. Additional permutation tests were carried out for the models that did not fulfill the normality assumption.

\section{Results}

\subsection{Pleasantness ratings of sweet and bitter samples}

The following section describes the results of the comparison of the VAS scale pleasantness ratings given by the participants for the sweet and bitter samples. The results of the paired sample t-test show that there was a significant difference in the pleasantness ratings between the sweet sample and the bitter sample $(t(88)=-17.44, p<0.001)$ with the bitter sample having lower pleasantness ratings (mean pleasantness 20.73 \pm 16.30 ) than the sweet (mean pleasantness $70.28 \pm 19.25$ ) one.

\subsection{ANS responses to taste and taste novelty (first block)}

A hundred and fifty-five participants completed the study. For this first block, the data of 22 participants were excluded from the analysis: 11 due to ectopic beats, two due to abnormal heart rhythms, one due to mistakes during the execution 
of the study, and eight participants due to movements during the measurement. In total, the data of 133 participants were included in the analysis. Forty-four were part of the water group used in a separate analysis. The remaining 89 participants were part of the analyses of taste novelty, taste, and the (dis)confirmation of expectations. From these, 49 were female $($ mean age $=24.4 \pm 4.2$, mean $\mathrm{BMI}=21.5 \pm 1.8)$ and 40 were male $($ mean age $=25.0 \pm 4.7$, mean $\mathrm{BMI}=22.5 \pm 2.4)$.

\subsubsection{Effect of taste novelty and taste on heart rate and skin conductance}

The following section describes the results of the analysis of the effect of taste (sweet vs bitter) and taste novelty (1st experience vs 2nd experience with a taste) on heart rate and skin conductance (see Fig. 3). The data of Group 1 and Group 2 were joined together given that the arrangement of the samples (order of bitter or sweet rounds) did not alter the results. Gender, age, and BMI were checked but not added in the final models, as they did not affect the main outcome of the model when included.

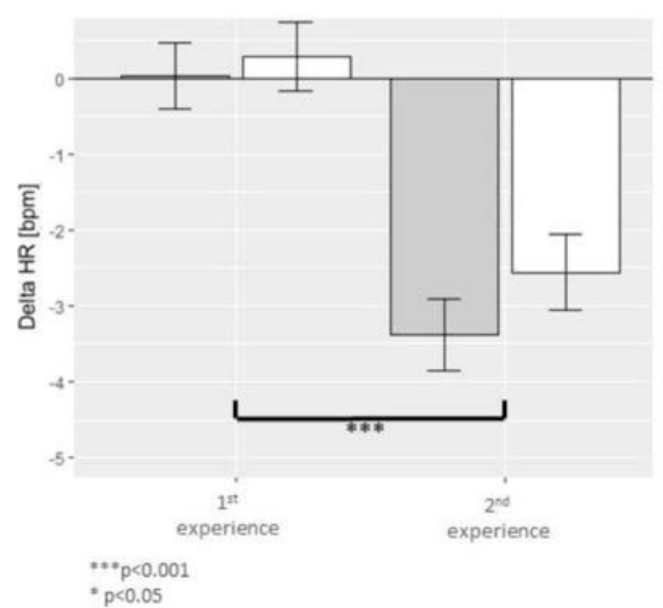

A)

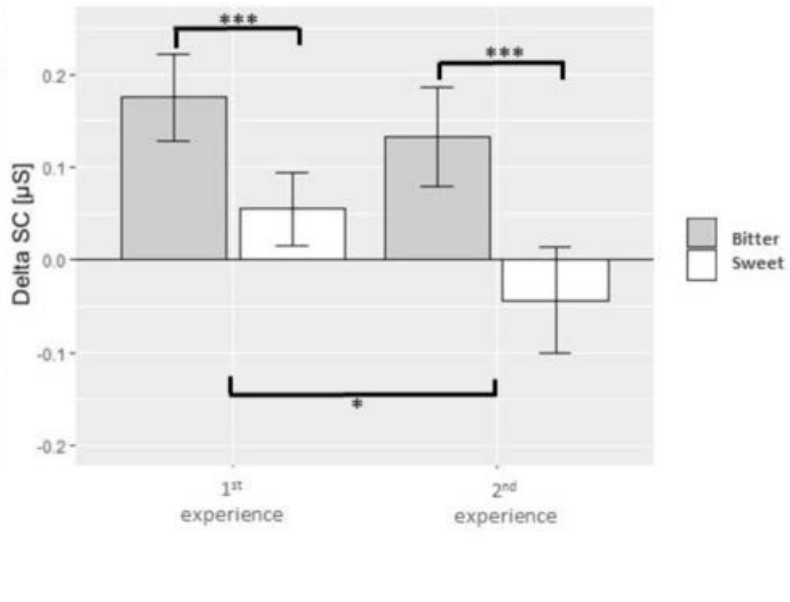

B)

Fig. 3 Mean ( \pm SE) of (A) delta heart rate (beats per minute) and (B) delta skin conductance ( $\mu$ s) during the first and second experience with the sweet and bitter samples $(\mathrm{N}=89)$.

\subsubsection{Heart rate}

There was a significant effect of taste novelty (1st vs 2 nd experience with a taste) in delta heart rate $(F(1,263)=78.69$, $p<0.001)$. Post hoc tests revealed that the differences between the first and second experience were found for both sweet and bitter, with the second experience presenting a larger decrease in heart rate than the first $(p<0.001)$. We did not find a main effect of taste $(F(1,284)=0.104, p=0.747)$ or of the interaction between taste and taste novelty $(F(1,263)=0.631$, $p=0.428$ ). These results show that, regardless of the taste, the second experience with a taste leads to a lower cardiac response than the first experience.

\subsubsection{Skin conductance}

There was a significant effect of taste novelty (1st vs 2nd experience with a taste) in delta skin conductance $(F(1,263)=4.36, p=0.038)$. Likewise, we found an effect of taste in delta skin conductance $(F(1,281)=26.84, p<0.001)$. There was no effect of the interaction between taste and taste novelty $(F(1,263)=0.68, p=0.412)$. Post hoc tests revealed that the second experience led to a smaller delta skin conductance than the first and that the bitter sample led to a larger increase in delta skin conductance than the sweet sample, which presented a negative delta skin conductance during the second tasting $(p<0.001)$.

\subsubsection{Analysis with water as neutral stimulus}

The following section describes the heart rate and skin conductance models assessing the novelty and taste effect for bitter, sweet, and water samples, done to complement the analysis of the first block of the study. See Fig. 4. 


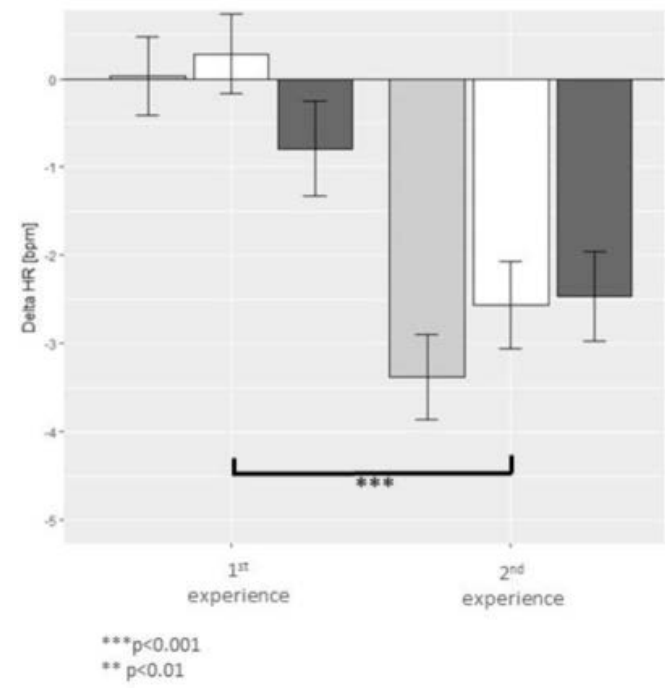

A)

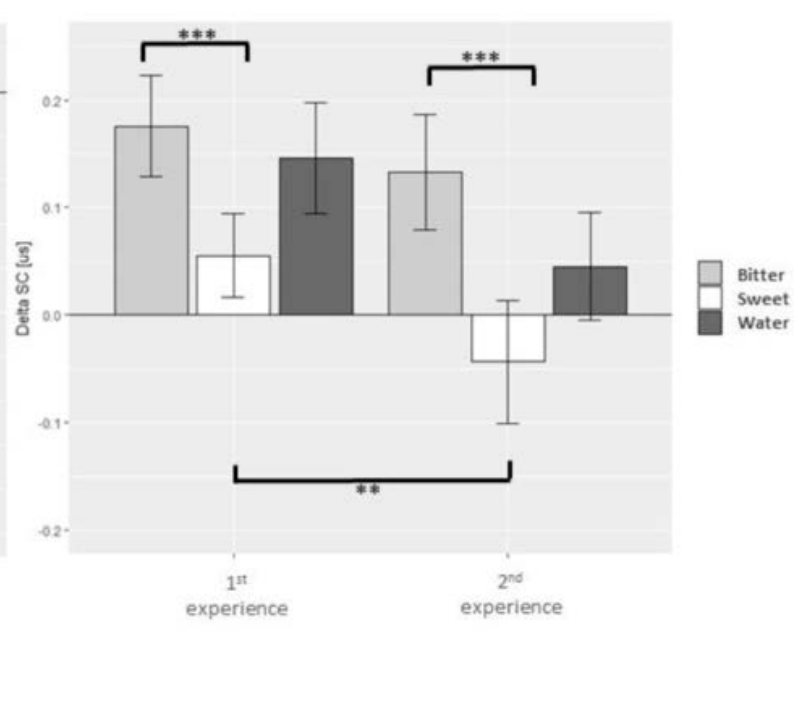

B)

Fig. 4 Mean $( \pm S E)$ of $(A)$ delta heart rate (beats per minute) and $(B)$ delta skin conductance ( $\mu$ s) during the first and second experience of the bitter, sweet and water samples $(N=133)$.

\subsubsection{Heart rate}

There was a significant effect of taste novelty (1st vs 2 nd experience with a taste) in delta heart rate $(F(1,398)=68.388$, $p<0.001)$. Post hoc tests revealed the second experience presented a larger decrease in heart rate than the first. We did not find a main effect of taste $(F(2,197)=0.99, p=0.370)$ or of the interaction between taste and taste novelty $(F(2,398)=2.56, p=0.078)$. These results show that the differences between the first experience and the second experience are seen for all samples, including that of water which had no taste.

\subsubsection{Skin conductance}

There was a significant effect of taste novelty (1st vs 2nd experience with a taste) in delta skin conductance $(F(1,398)=8.43, p=0.268)$. Likewise, we found an effect of taste $(F(2,197)=9.39, p<0.001)$. Post hoc tests revealed that the second experience led to smaller increases in delta skin conductance than the first. The sweet and bitter samples led to different changes in delta skin conductance, with the bitter sample leading to larger increases in skin conductance than those of the sweet. The delta skin conductance of the water sample, however, did not differ from those of the sweet and bitter samples. We did not find an effect of the interaction between taste and taste novelty $(F(2,398)=0.463, p=0.412)$. These results show that the effect of taste novelty is also present in the water sample and that the skin conductance of the water samples remains similar to that of the sweet and bitter samples.

\subsection{Effect of the (dis)confirmation of expectations on ANS responses (second block)}

From the 155 participants that completed the study, the data of 18 were excluded from the analysis of this second block. Eleven were excluded due to ectopic beats, two due to abnormal heart rhythms, one due to mistakes during the execution of the study, and four participants due to movements during the measurement. In total, the data of 137 participants was analyzed. From these, 26 were used for a separate analysis and given only water. The remaining 111 were used for the analysis of the disconfirmation of expectations. The demographic information is shown on Table 1.

Table 1 Demographics of the sample by divided by group $(N=137)$. 


\begin{tabular}{|c|c|c|c|c|c|c|}
\hline & $\begin{array}{l}\text { Bitter expected } \\
\text { first }\end{array}$ & $\begin{array}{l}\text { Bitter unexpected } \\
\text { first }\end{array}$ & $\begin{array}{l}\text { Sweet expected } \\
\text { first }\end{array}$ & $\begin{array}{l}\text { Sweet unexpected } \\
\text { first }\end{array}$ & Water & $\begin{array}{l}\mathrm{P} \\
\text { value }\end{array}$ \\
\hline$N$ & 24 & 28 & 29 & 30 & 26 & - \\
\hline $\begin{array}{l}\text { Gender: Female } \\
\text { (Male) }\end{array}$ & $15(9)$ & $16(12)$ & 18(11) & 15(15) & $14(12)$ & $0.865 a$ \\
\hline Age (years) & $25.1 \pm 3.8$ & $22.8 \pm 3.3$ & $25.5 \pm 3.9$ & $24.6 \pm 5.4$ & \multicolumn{2}{|c|}{$25.8 \pm 4.30 .074 b$} \\
\hline BMI (kg/m2) & $22.2 \pm 2.3$ & $21.6 \pm 2.0$ & $21.4 \pm 1.7$ & $22.4 \pm 1.9$ & \multicolumn{2}{|c|}{$21.9 \pm 2.40 .341 b$} \\
\hline
\end{tabular}

ap-value calculated with chi square test.

bp-value calculated with anova.

\subsubsection{Effect of (dis)confirmation of expectations on heart rate and skin conductance}

The following section describes the results of the analysis of the effect of tasting samples with a taste that confirms (expected) and disconfirms expectations (unexpected) on heart rate and skin conductance (See Fig. 5). The data of Group 1 and Group 2 and that of Group 3 and 4 were joined together given that the arrangement of the samples (confirmation or disconfirmation first) did not alter the results. Gender, age, and BMI were not added in the models as they did not affect the main outcome when included.

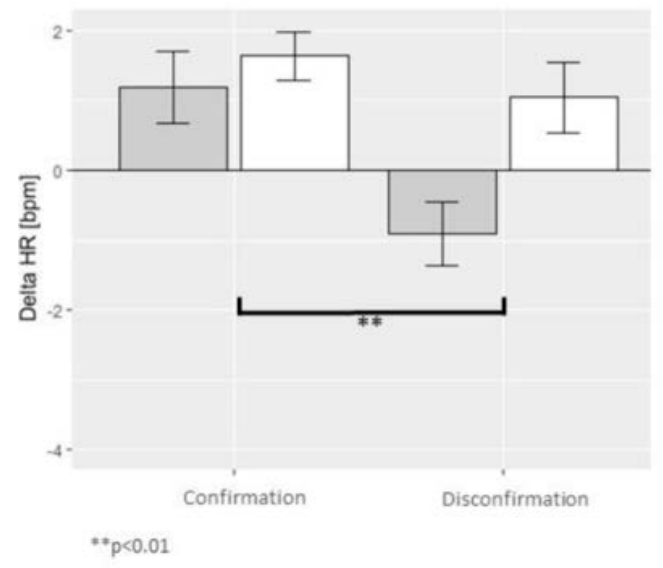

A)

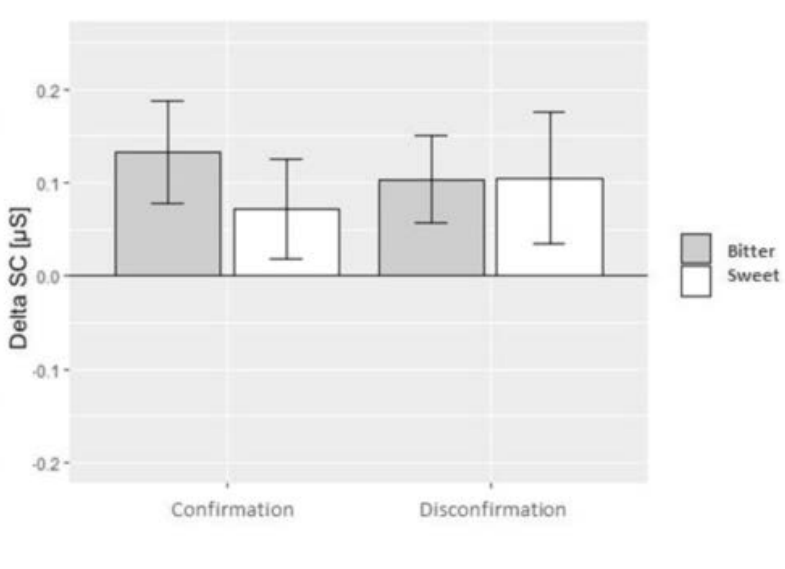

B)

Fig. 5 Mean $( \pm S E)$ of $(A)$ delta heart rate (beats per minute) and $(B)$ delta skin conductance $(\mu s)$ during the tasting of samples that confirm (expected) and disconfirm (unexpected) expectations $(N=111)$. The tastes shown on the graphs are the real tastes of the samples given to participants. Note that the effect of taste for heart rate was significant but not possible to represent in the graph.

\subsubsection{Heart rate}

There was a significant effect of disconfirmation on delta heart rate $(F(1,218)=8.75, p=0.003)$. Likewise, the effect of the real taste was significant $(F(1,218)=6.98, p=0.009)$. Posthoc tests revealed that samples with tastes that disconfirmed expectations led to a decrease in heart rate and that the bitter sample led to a larger decrease in heart rate that the sweet sample. However, there was no effect of the interaction between real taste and disconfirmation $(F(1,218)=2.76, p=0.098)$.

\subsubsection{Skin conductance}

Disconfirmation did not have an effect on skin conductance $(F(1,218)=0.01, p=0.977)$. Likewise, the effects of taste and that of the interaction between taste and disconfirmation were not significant $(F(1,218)=0.28, p=0.600$ and $F(1,218)=0.31, p=0.580$ respectively). These results suggest that skin conductance does not change between tastes that confirm and disconfirm expectations as presented in this study. 


\subsubsection{Analyses including water as neutral stimulus}

The following section describes the heart rate and skin conductance models used for the analysis used to corroborate the results of the second part of the study. As only the data of the samples that confirmed expectations and that were given in the second position was used, the results shown here relate to the responses of 79 participants. See Fig. 6.

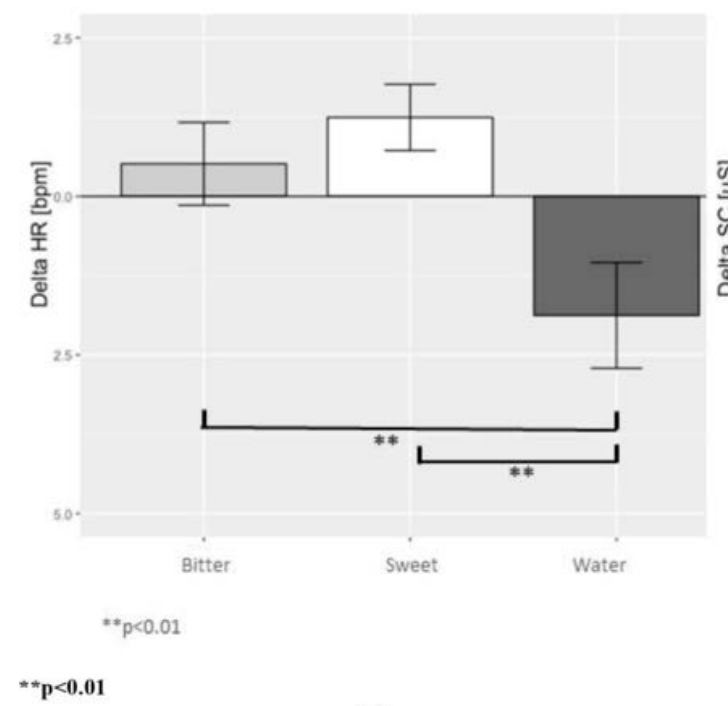

A)

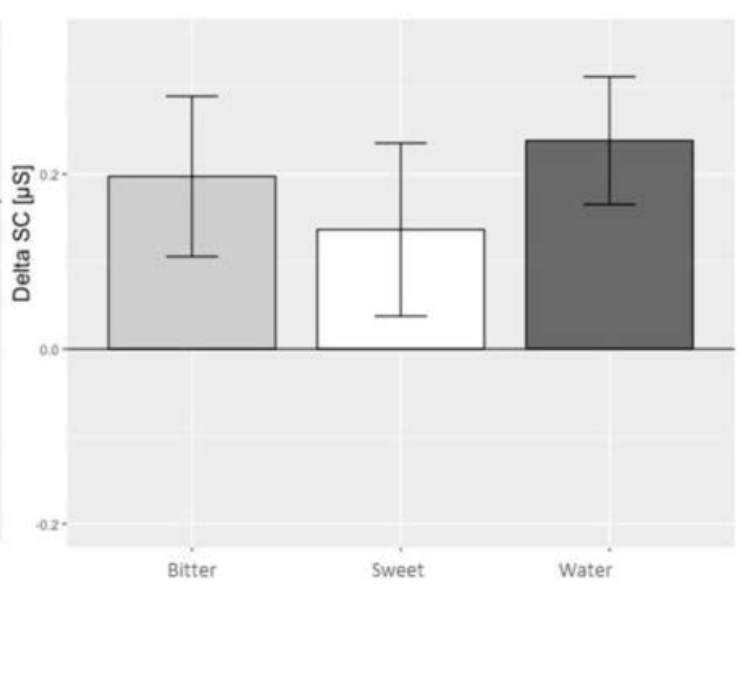

B)

Fig. 6 Mean $( \pm S E)$ of $(A)$ delta heart rate (beats per minute) and (B) delta skin conductance ( $\mu$ s) during the tasting of the first samples that confirm expectations $(\mathrm{N}=79)$.

\subsubsection{Heart rate}

There was a significant effect of real taste on delta heart rate $(F(2,76)=5.97, p=0.004)$. The main differences were between the bitter taste and water, and between the sweet taste and water. These results show that the delta heart rate is similar for the sweet and bitter samples but is different from that of the water samples.

\subsubsection{Skin conductance}

The effect of real taste on delta skin conductance was not significant $(F(2,76)=0.34, p=0.711)$. These results show that the delta skin conductance remains similar for the water samples and the samples with the bitter and sweet taste.

\section{Discussion}

The aim of this study was to determine the differences in ANS responses associated with taste novelty (first experience with a taste), disconfirmation of expectations and the differences associated to valence (taste with a positive valence vs taste with a negative valence). One of the main findings of this study is that heart rate seems to be more sensitive to the differences related to taste novelty and the disconfirmation of expectations while skin conductance is sensitive to changes related to novelty and valence but not to the disconfirmation of expectations.

Taste novelty led to differences in heart rate, regardless of the valence of the taste. While heart rate remained similar to baseline for the first experience, the second experience with the samples led to a cardiac deceleration. Cardiac deceleration is a characteristic of the orientation response (OR). It occurs when dishabituation has taken place. Before such deceleration, however, the immediate response is that of arousal and is accompanied by a brief cardiac acceleration and a change in skin conductance. Longer lasting responses, signaled by cardiac deceleration, immediately follow and are associated to responses to attentional and sensory processing (Lacey \& Lacey, 1970; Pribram, 1979). It seems likely that the heart rate responses during the first experience are a result of the combination of both arousal and attentional processing. The brief cardiac acceleration related to the arousal response to the novelty of the taste masked the expected deceleration of the OR and as a result the heart rate remained close to baseline. Only during the second experience with the samples, when the taste was no longer novel and the brief acceleration was no longer present, cardiac deceleration was the main response. 
Skin conductance responses differed according to taste novelty and also to valence. The first experience with a taste led to larger increases in skin conductance than the second experience. Stimuli that lead to higher skin conductance responses include novel, significant, or intense stimuli (Dindo \& Fowles, 2008). Even for novel stimuli with moderate or low intensity, an increase in skin conductance after its presentation is interpreted as an attention or orientation response. This response indicates that the novel stimulus has been detected (Vila, 2004). Hence, the skin conductance changes to novelty are, same as the cardiac responses, related to the OR to the first encounter with the taste.

The skin conductance changes related to taste valence were stronger than those related to taste novelty. This might be because skin conductance primarily reflects the activation of the sympathetic nervous system. While an OR leads to a sympathetic-parasympathetic coactivation, effort mobilizing aspects, such as a defense mechanism, lead solely to sympathetic activation (Kreibig, 2016). In our study the bitter taste, which was negative, led to an increase in skin conductance while the sweet taste, which was positive, led to a decrease. The inner connotations of the bitter taste, constantly associated to noxious or toxic substances, might have triggered a strong defensive response which resulted in the increase in skin conductance (Rousmans et al., 2000). The exact mechanism why the sweet sample gives weaker responses is still unknown, Rousmans et al. (2000) mentioned that it may be due to the fact that the sweet taste is familiar and frequently consumed and hence, the weak responses might be a product of habituation to the taste.

The results of the second block show that the disconfirmation of expectations was associated to changes in heart rate but not to skin conductance. Contrary to what we hypothesized, samples that disconfirmed expectations led to lower heart rates than those that confirmed expectations. This was seen particularly in the cases in which participants expected a positive taste (sweet) but got a negative one (bitter). It is possible that, instead of capturing the defense mechanism to the change in taste, the heart rate responses are again representing an orienting response to this change/disconfirmation ("this is not the taste I was told") as such change is deserving of attention (Graham \& Clifton, 1966). An interesting finding was that heart rate responses were only sensitive to taste in the second part of the study but not in the first. This may be due to the tasting of samples that disconfirmed expectations included in the second part. The reactivity to the stimulus was heightened and the attention of the participants became stronger due to the significance of the change/disconfirmation in taste (Bradley, Keil, \& Lang, 2012).

The analysis with water done with the data from the first block of the study confirms our interpretations regarding the ANS responses to taste novelty and taste. Heart rate and skin conductance responses were different between the first and the second tasting for all samples. Hence, we can confirm that novelty in itself leads to differences in these ANS responses. In the case of the effect of taste, the analysis with the water sample finds again that the heart rate responses do not differ in terms of taste. The skin conductance responses showed that the sweet taste had the lowest skin conductance while the bitter the highest. The changes in skin conductance related to water remained between those of the sweet and bitter samples. Only the difference between the sweet and bitter samples was statistically significant. It seems, therefore, that skin conductance can only differentiate between tastes of opposite valence. The analysis with water done with the data from the second block of the study shows that heart rate increased for the sweet and bitter samples while the water sample showed a low decrease. These heart rate and skin conductance responses were different from those of the first block of the study. Though in both cases the samples are congruent, the analyses performed are different as is the design and sample size. This may account for the differences found.

While the findings on ANS and taste novelty match our hypothesis, some of the results on valence were different than expected. The skin conductance responses to valence match what has been reported in other studies that use basic tastes (Horio, 2000; Robin, Rousmans, Dittmar, \& Vernet-Maury, 2003; Rousmans et al., 2000), but the same does not apply for our results regarding heart rate. The findings of previous studies state that the tasting of a sample leads to an increase in heart rate, with quinine among those that give the strongest heart rate responses and sucrose the weakest. Our study found that the tasting of samples led to a cardiac deceleration and that this deceleration was similar for sweet and bitter samples. An increase was only seen in the second block of the study, where expectations were disconfirmed. However, in this part the heart rate responses to the sweet sample were stronger than those to the bitter one. Whether these differences were related again to the influence of the orientating response related to the disconfirmation (with bitter samples leading to a stronger orientating response and, as a consequence, a stronger cardiac deceleration) is still unclear.

The findings on ANS and the disconfirmation of expectations present some similarities and differences from those of a previous study (Verastegui-Tena et al., 2017) which also dealt with the topic of the disconfirmation of expectations. Both studies found an increase in skin conductance for the negative stimuli that disconfirmed expectations, and this increase did not differ significantly from that of the positive one. However, while in the previous study both the positive and negative stimuli that disconfirmed expectations led to a similar decrease in heart rate, in this study only the negative stimuli led to 
a decrease and it differed significantly from that of the positive. It is possible that this difference is due to the stimuli used in the studies. The stimuli in Verastegui-Tena et al. were images while the present study used samples with different tastes. Moreover, it did not separate the effect of novelty and valence from that of the disconfirmation of expectations while this study looks at these effects separately.

Our study has certain limitations. While we have data on perceived taste intensity for the first block of the study (effect of taste and taste novelty), we do not have this information for the second block (effect of the (dis)confirmation of expectations). This might have played a role in the absence of changes in skin conductance related to the disconfirmation of expectations as skin conductance has been said to be related to stimulus intensity (Bensafi et al., 2002; Dawson, Schell, \& Filion, 2000). Moreover, the first block of the study showed a significant effect of the taste intensity covariate on skin conductance. It is possible, therefore, that in order for skin conductance to discriminate among taste stimuli it is necessary to include the data about the perceived intensity. This is further strengthened by the results of the analysis with water of the first block. The participants that tasted only water were not asked to rate the pleasantness and taste intensity of the water sample as it was assumed to be neutral. As a consequence, it was not possible to add these data in the analysis and we could only find differences between the extremes of the valence scale (sweet vs bitter samples). The data on taste intensity might have helped find differences between the water sample and the samples with taste. While an analysis on latency and amplitude may have also been helpful to find some differences, the short time intervals between stimuli may not allow it. Participants may not have gone back to baseline before being presented with another stimulus. Lastly, our study focuses on the effects of stimuli that are opposite in valence and congruity. Hence, we cannot know if the same effects would be found if such contrast were not present.

The present work brings more insight into the ANS responses to taste novelty, taste valence, and the disconfirmation of expectations. It looks at the effects of these appraisals on ANS responses and interprets their differences. Moreover, it shows that both heart rate and skin conductance capture changes related to novelty but that, while heart rate can also capture the differences related to the disconfirmations of expectations, the same does not apply for skin conductance. Likewise, it shows that skin conductance, though capable of capturing both novelty and valence, gives clearer results when measuring taste valence. The findings have implications for future research as they show that some ANS responses might be more useful for a particular design than for others. For example, skin conductance and other markers of sympathetic response could be used for products that contrast in particular dimensions such as valence and arousal while heart rate might be helpful to measure the habituation to novel products. Future studies should look further into the appropriate use of ANS responses for each design.

\section{Conclusion}

The aim of the present work was to evaluate the differences in heart rate and skin conductance related to taste novelty, valence and the disconfirmation of expectations. To the best of our knowledge, this is the first study to look separately at the effect of these appraisals on ANS responses. Our results show that cardiac responses differ in regards to novelty and the disconfirmation of expectations. Skin conductance responses differ in regards to novelty and valence but they do not change in regards to the disconfirmation of expectations. The changes in ANS responses in this study may be related to different mechanisms. Changes to novelty and the disconfirmation of expectations seem to be related to the orientation response while the changes to valence may be capturing defense mechanisms. Further research is, however, necessary to confirm these interpretations.

\section{Acknowledgements:}

The authors would like to thank Aurelia Schulte-Holierhoek Msc. for her support during the execution of the study.

\section{References}

Bartoszek and Cervone, 2016Bartoszek G. and Cervone D., Toward an implicit measure of emotions: Ratings of abstract images reveal distinct emotional states, Cognition and Emotion 2016, 1-15. 
Bensafi et al., 2002Bensafi M., Rouby C., Farget V., Bertrand B., Vigouroux M. and Holley A., Autonomic nervous system responses to odours: The role of pleasantness and arousal, Chemical Senses 27 (8), 2002, 703-709.

Bradley et al., 2012Bradley M.M., Keil A. and Lang P.J., Orienting and emotional perception: Facilitation, attenuation, and interference, Frontiers in Psychology 3, 2012, 493.

Danner et al., 2014Danner L., Haindl S., Joechl M. and Duerrschmid K., Facial expressions and autonomous nervous system responses elicited by tasting different juices, Food Research International 64, 2014, 81-90.

Dawson et al., 2000Dawson M.E., Schell A.M. and Filion D.L., The electrodermal system, In: Cacioppo J.T., Tassinary L.G. and Berntson G.G., (Eds.), Handbook of psychophysiology, 2000, Cambridge University Press; New York, New York US.

de Geus et al., 1995de Geus E.J.C., Willemsen G.H.M., Klaver C.H.A.M. and van Doornen L.J.P., Ambulatory measurement of respiratory sinus arrhythmia and respiration rate, Biological Psychology 41 (3), 1995, 205-227.

de Wijk and Boesveldt, 2016de Wijk R.A. and Boesveldt S., Responses of the autonomic nervous system to flavours, In: Multisensory flavor perception: From fundamental neuroscience through to the marketplace, 2016, Woodhead Publishing.

de Wijk et al., 2012de Wijk R.A., Kooijman V., Verhoeven R.H.G., Holthuysen N.T.E. and de Graaf C., Autonomic nervous system responses on and facial expressions to the sight, smell, and taste of liked and disliked foods, Food Quality and Preference 26 (2), 2012, 196-203.

Delplanque et al., 2009Delplanque S., Grandjean D., Chrea C., Coppin G., Aymard L., Cayeux I., et al., Sequential unfolding of novelty and pleasantness appraisals of odors: Evidence from facial electromyography and autonomic reactions, Emotion 9 (3), 2009, 316-328.

Derryberry and Reed, 2002Derryberry D. and Reed M., Information processing approaches to individual differences in emotional reactivity, In: Davidson R.J., Scherer K.R. and Goldsmith H.H., (Eds.), Handbook of affective sciences, 2002, Oxford University Press.

Dindo and Fowles, 2008Dindo L. and Fowles D.C., The skin conductance orienting response to semantic stimuli: Significance can be independent of arousal, Psychophysiology 45 (1), 2008, 111-118.

Ellsworth and Scherer, 2002Ellsworth P.C. and Scherer K.R., Appraisal processes in emotion, In: Davidson R.J., Scherer K.R. and Goldsmith H.H., (Eds.), Handbook of affective sciences, 2002, Oxford University Press, USA; New York, NY.

Frijda and Mesquita, 1998Frijda N.H. and Mesquita B., The analysis of emotions: Variations of dimensions, In: Mascolo M.F. and Griffin S., (Eds.), What develops in emotional development? 1998, Plenum Press.

Graham and Clifton, 1966Graham F.K. and Clifton R.K., Heart-rate change as a component of the orienting response, Psychological Bulletin 65 (5), 1966, 305-320.

He et al., 2016He W., Boesveldt S., de Graaf C. and de Wijk R.A., The relation between continuous and discrete emotional responses to food odors with facial expressions and non-verbal reports, Food Quality and Preference 48, 2016, 130-137.

Horio, 2000Horio T., Effects of various taste stimuli on heart rate in humans, Chemical Senses 25 (2), 2000, $149-153$.

Kreibig, 2016Kreibig S., Autonomic nervous system aspects of positive emotions, In: Tugade M.M., Shiota M.N., Kirby L.D. and Fredrickson B.L., (Eds.), Handbook of positive emotions, 2016, Guilford Publications.

Lacey and Lacey, 1970Lacey J.I. and Lacey B.C., Some autonomic-central nervous system interrelationships, In: Black P., (Ed), Physiological correlates of emotion, 1970, Academic Press; New York.

Lazarus and Folkman, 1984Lazarus R.S. and Folkman S., Situation factors influencing appraisal, In: Stress, appraisal, and coping, 1984, Springer Publishing Company.

Lebens et al., 2011Lebens H., Roefs A., Martijn C., Houben K., Nederkoorn C. and Jansen A., Making implicit measures of associations with snack foods more negative through evaluative conditioning, Eating Behaviors 12 (4), 2011, 249253.

Levenson, 2014Levenson R.W., The autonomic nervous system and emotion, Emotion Review 6 (2), 2014, $100-112$. 
Levine et al., 2012Levine M.S., Ramchandani P. and Rubesin S.E., Practical fluoroscopy of the GI and GU tracts, 2012, Cambridge University Press; Cambridge.

Mathôt et al., 2012Mathôt S., Schreij D. and Theeuwes J., OpenSesame: An open-source, graphical experiment builder for the social sciences, Behavior Research Methods 44 (2), 2012, 314-324.

Mauss and Robinson, 2009Mauss I.B. and Robinson M.D., Measures of emotion: A review, Cognition \& Emotion 23 (2), 2009, 209-237.

McCorry, 2007McCorry L.K., Physiology of the autonomic nervous system, American Journal of Pharmaceutical Education 71 (4), 2007.

Mojet et al., 2015Mojet J., Dürrschmid K., Danner L., Jöchl M., Heiniö R.-L., Holthuysen N., et al., Are implicit emotion measurements evoked by food unrelated to liking?, Food Research International 76, 2015, 224-232.

Moors et al., 2013Moors A., Ellsworth P.C., Scherer K.R. and Frijda N.H., Appraisal theories of emotion: State of the art and future development, Emotion Review 5 (2), 2013, 119-124.

Pribram, 1979Pribram K., The orienting reaction: Key to brain representational mechanisms, In: Kimmel H.D., van Olst E.H., Orlebeke J.F. and Division N.A.T.O.S.A., (Eds.), The orienting reflex in humans: An international conference sponsored by the scientific affairs division of the north atlantic treaty organization, leeuwenhorst congress center, the netherlands, June 1978 L 1979, Erlbaum Associates.

Robin et al., 2003Robin O., Rousmans S., Dittmar A. and Vernet-Maury E., Gender influence on emotional responses to primary tastes, Physiology \& Behavior 78 (3), 2003, 385-393.

Rousmans et al., 2000Rousmans S., Robin O., Dittmar A. and Vernet-Maury E., Autonomic nervous system responses associated with primary tastes, Chemical Senses 25 (6), 2000, 709-718.

Spinelli and Niedziela, 2016Spinelli S. and Niedziela M., 5 - emotion measurements and application to product and packaging development A2 - burgess, peter, Integrating the packaging and product experience in food and beverages, 2016, Woodhead Publishing.

Tarancón et al., 2014Tarancón P., Sanz T., Fiszman S. and Tárrega A., Consumers' hedonic expectations and perception of the healthiness of biscuits made with olive oil or sunflower oil, Food Research International 55, 2014, 197206.

Verastegui-Tena et al., 2017Verastegui-Tena L., Schulte-Holierhoek A., van Trijp H. and Piqueras-Fiszman B., Beyond expectations: The responses of the autonomic nervous system to visual food cues, Physiology \& Behavior 179, 2017, 478-486.

Vila, 2004Vila J., Psychophysiological assessment A2 - spielberger, In: Charles D., (Ed), Encyclopedia of Applied Psychology 2004, Elsevier; New York.

Walsh et al., 2017Walsh A.M., Duncan S.E., Bell M.A., O'Keefe S.F. and Gallagher D.L., Breakfast meals and emotions: Implicit and explicit assessment of the visual experience, Journal of Sensory Studies 32 (3), 2017, e12265-n/a. 